\title{
Phosphatase WIP1 regulates adult neurogenesis and WNT signaling during aging
}

\author{
Yunhua Zhu, ${ }^{1}$ Oleg N. Demidov, ${ }^{2}$ Amanda M. Goh, ${ }^{3}$ David M. Virshup, ${ }^{4}$ \\ David P. Lane, ${ }^{3}$ and Dmitry V. Bulavin ${ }^{1,5}$

\begin{abstract}
${ }^{1}$ Institute of Molecular and Cell Biology, Proteos, Singapore. 2INSERM U866, University of Burgundy, Dijon, France. ${ }^{3}$ p53 Laboratory, Agency for Science, Technology and Research (A*STAR), Institute of Medical Biology (A*STAR), Singapore. ${ }^{4}$ Program in Cancer and Stem Cell Biology, Duke-National University of Singapore Graduate Medical School, Singapore. 5Institute for Research on Cancer and Ageing of Nice, INSERM, U1081-UMR CNRS 7284, University of Nice - Sophia Antipolis, Centre Antoine Lacassagne, Nice, France.
\end{abstract}

\begin{abstract}
The number of newly formed neurons declines rapidly during aging, and this decrease in neurogenesis is associated with decreased function of neural stem/progenitor cells (NPCs). Here, we determined that a WIP1-dependent pathway regulates NPC differentiation and contributes to the age-associated decline of neurogenesis. Specifically, we found that WIP1 is expressed in NPCs of the mouse subventricular zone (SVZ) and aged animals with genetically enhanced WIP1 expression exhibited higher NPC numbers and neuronal differentiation compared with aged WT animals. Additionally, augmenting WIP1 expression in aged animals markedly improved neuron formation and rescued a functional defect in fine odor discrimination in aged mice. We identified the WNT signaling pathway inhibitor DKK3 as a key downstream target of WIP1 and found that expression of DKK3 in the SVZ is restricted to NPCs. Using murine reporter strains, we determined that DKK3 inhibits neuroblast formation by suppressing WNT signaling and Dkk3 deletion or pharmacological activation of the WNT pathway improved neuron formation and olfactory function in aged mice. We propose that WIP1 controls DKK3-dependent inhibition of neuronal differentiation during aging and suggest that regulating WIP1 levels could prevent certain aspects of functional decline of the aging brain.
\end{abstract}

\section{Introduction}

New neurons are generated continuously in 2 distinct regions of the mammalian brain, the subventricular zone (SVZ) and subgranular zone (SGZ). The SVZ represents the largest reserve of neural stem cells (NSCs) in mice and can be identified by staining with NESTIN, SOX2, and GFAP. NSCs are largely quiescent but occasionally enter the cell cycle to divide asymmetrically to generate another NSC plus a transit-amplifying progenitor. Transit-amplifying cells undergo rapid symmetric divisions to give rise to neuroblasts that express polysialylated neuronal cell adhesion molecule (PSA-NCAM) and doublecortin (DCX). In mice, DCX-positive neuroblasts migrate through the rostral migratory stream toward the olfactory bulb (OB), in which they differentiate further, integrate into the existing neuronal network, and contribute to various olfactory functions, including fine odor discrimination.

Adult neurogenesis declines rapidly during aging, resulting in functional decline of the brain. One important aspect of this aging process is the reduced formation of new neurons that coincides with a reduction in the number of neural stem/progenitor cells (NPCs). However, it is not entirely clear whether increasing the number of stem cells is sufficient for improving new neuron production; notably, multiple aging-related changes could account for a neuron deficit in the OB (1). Attenuation of neuronal commitment of NPCs is one such mechanism that can contribute

Conflict of interest: The authors have declared that no conflict of interest exists.

Note regarding evaluation of this manuscript: Manuscripts authored by scientists associated with Duke University, The University of North Carolina at Chapel Hill, Duke-NUS, and the Sanford-Burnham Medical Research Institute are handled not by members of the editorial board but rather by the science editors, who consult with selected external editors and reviewers.

Citation for this article: J Clin Invest. 2014;124(7):3263-3273. doi:10.1172/JCI73015 to aging-related changes in adult neurogenesis. Increasing evidence supports the idea that, during aging, WNT signaling has an instructive role in neuronal fate determination from neural progenitors (2). For example, WNT3 expression decreases during aging (3), while levels of the WNT antagonist Dickkopf 1 (DKK1) increase specifically in the hippocampus (4). It remains unclear whether WNT plays any role in the SVZ; if it does, the mechanism for its regulation during aging is largely unknown.

In a previous study, we identified WT $\mathrm{p} 53$-induced phosphatase 1 (WIP1) as an important molecular component of adult neurogenesis in the SVZ (5). WIP1 maintains new neuron formation in a p53-dependent manner through regulation of cell cycle progression and neurogenesis. In the current study, we extended our analysis to investigate the significance of WIP1 regulation in a physiologically relevant context. We found that WIP1 is specifically expressed in SVZ NPCs and its expression decreases with aging, contributing to the regulation of adult neurogenesis through DKK3. Expression of DKK3 appeared to be restricted to SVZ NPCs and was absent in the SGZ. We also found that DKK3 inhibited canonical WNT signaling and WNT-induced neurogenesis. In turn, overexpression of WIP1 repressed DKK3, rescuing a neuron formation defect in the $\mathrm{OB}$ of aged mice.

\section{Results}

WIP1 is expressed in mouse NPCs and controls p53 activity during aging. As WIP1 is essential in NPC functions (5), we investigated its expression in the SVZ. We first analyzed a reporter line in which a $\beta$-gal (Wip1$\beta$-gal) gene was knocked in in the endogenous mouse Wip1 locus. Consistent with endogenous expression of WIP1 (6), $\beta$-gal staining was high in the testes of knock-in reporter mice, while it was absent in WT controls (Supplemental Figure 1A; supplemental material available online with this article; doi:10.1172/JCI73015DS1). In the 
A

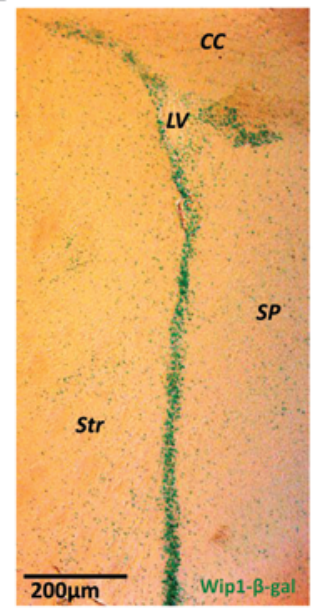

E

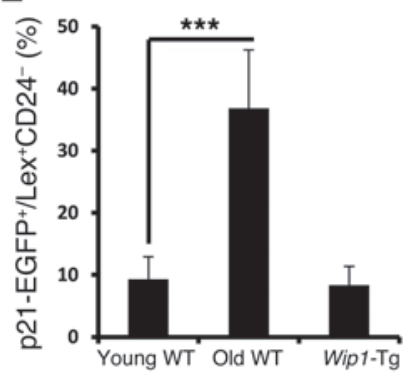

B Nestin/Wip1-8-gal/DAPI

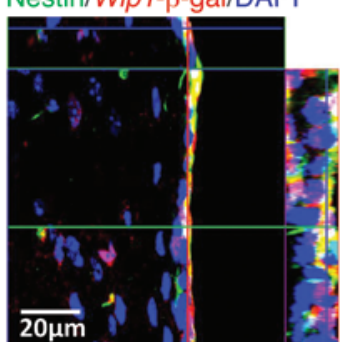

PSA-NCAM/Wip1- $\beta$-gal/DAPI

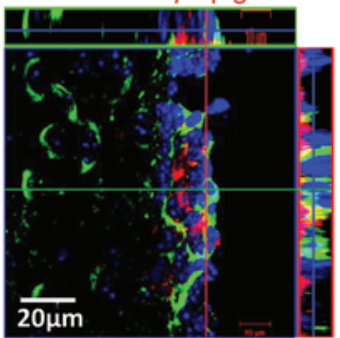

F

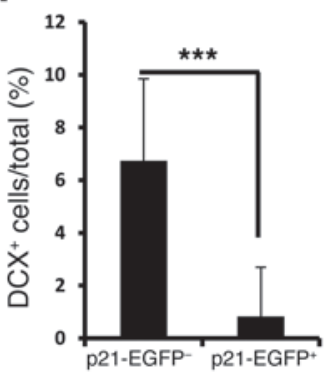

MASH1/Wip1- $\beta$-gal/DAPI

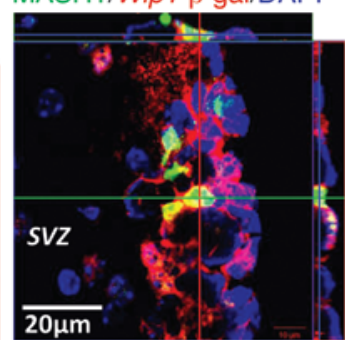

$\mathrm{S} 100 \mathrm{~B} / \mathrm{W} / \mathrm{H}$

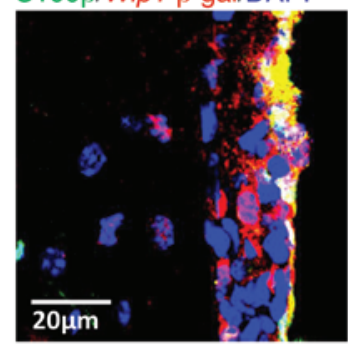

C
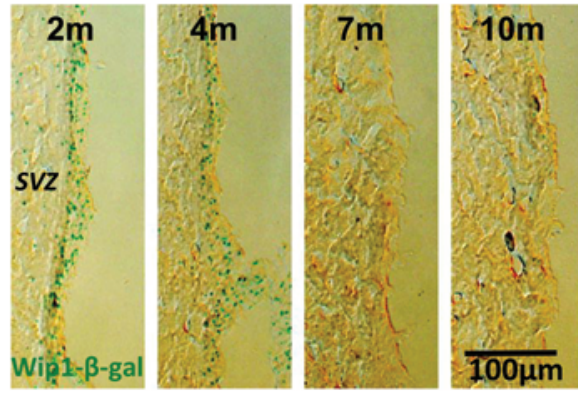

D

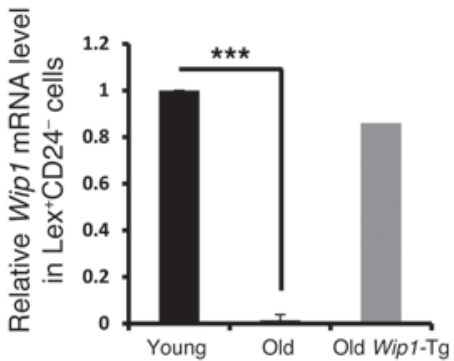

G

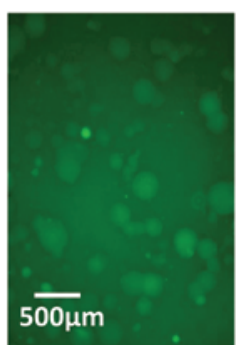

2 mo WT

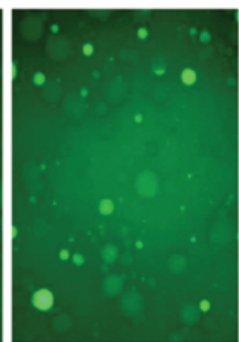

9 mo WT
H

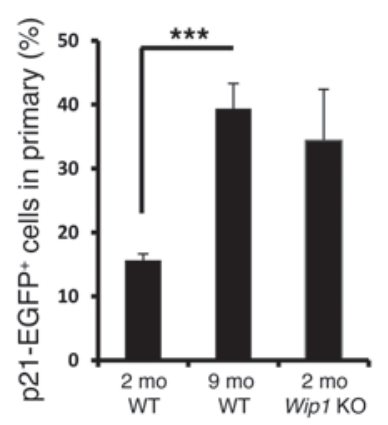

Figure 1

Expression of WIP1 in NSCs and p53 activation during aging. (A) Representative image of $\beta$-gal staining of brains of Wip1- $\beta$-gal mice. LV, lateral ventricle; CC, corpus callosum; Str, stratum; SP, septum. (B) Representative photographs of colocalization analysis of Wip1- $\beta$-gal and NESTIN, MASH1, PSA-NCAM, and S100 $\beta$. Note Wip1- $\beta$-gal expression in NSCs and transit-amplifying cells but not in neuroblasts. (C) Representative images of Wip1- $\beta$-gal in the SVZ regions of 2-, 4-, 7-, and 10-month-old mice. (D) Real-time PCR analysis of Wip1 mRNA in sorted LeX+CD24SVZ cells from 2- to 3-month-old (young) and 1-year-old (old) WT mice as well as from 1-year-old Wip1-Tg mice. The gate for sorting is shown in Supplemental Figure 1D. Data are mean \pm SD. (E) Analysis of EGFP signals from p21 promoter in LeX+CD24- SVZ cells obtained from 2- to 3-month-old and 1-year-old WT mice as well as from 1-year-old Wip1-Tg mice. Detailed analysis is shown in Supplemental Figure 1G. Data are mean \pm SEM. (F) Quantification of percentages of DCX-positive cells after 2 days differentiation for p21-EGFP-positive and -negative cells. Data are mean \pm SD. (G) Representative images of primary NSPs isolated from 2- and 9-month-old p21-EGFP reporter mice. (H) Quantification of the percentages of p21-EGFP-positive cells in primary neurospheres isolated from 2- and 9-month-old WT mice and 2-month-old Wip1 KO mice. Data are mean \pm SEM. ${ }^{\star \star \star} P<0.005$. Scale bar: $200 \mu \mathrm{m}(\mathbf{A}) ; 20 \mu \mathrm{m}(\mathbf{B}) ; 100 \mu \mathrm{m}(\mathbf{C}) ; 500 \mu \mathrm{m}(\mathbf{G})$.

brain, WIP1 expression was observed in the subventricular area (Figure $1 \mathrm{~A}$ ), but very little was observed in the $\mathrm{OB}$ or rostral migratory stream (Supplemental Figure 1, B and C). Analysis of $\beta$-gal-positive cells revealed coexpression with NESTIN and MASH1 but little with PSA-NCAM (Figure 1B). This suggests that WIP1 is primarily expressed in NPCs but not in neuroblasts. Furthermore, we found an age-dependent decline in Wip1- $\beta$-gal activity (Figure 1C). In order to confirm WIP1 expression in NPCs, we isolated $\mathrm{LeX}^{+} \mathrm{CD} 24^{-}$cells that are enriched for NSCs (refs. 7, 8, and Supplemental Figure 1D) and used a real-time PCR to analyze Wip1 mRNA expression in different populations of SVZ cells. We detected Wip1 mRNA expression in the $\mathrm{LeX}^{+} \mathrm{CD} 24^{-}$population, while its expression was almost completely diminished with aging (Figure 1D).
WIP1 regulates NPCs via p53 (5), so next we investigated p53 activity in vivo. For these experiments, we used a transgenic reporter mouse line that carries an EGFP under the control of a p53 response element from the $p 21^{\text {Cip }}$ promoter ( $p 21$-EGFP) (9). There was an EGFP signal in neurospheres (NSPs) from WT mice but not from $p 53 \mathrm{KO}$ mice, confirming the specificity of the reporter gene (Supplemental Figure 1E). We crossed $p 21$-EGFP reporter mice with Wip1 KO mice and confirmed that NSPs from Wip1 KO mice contained a higher percentage of $p 21$-EGFP-positive cells (Supplemental Figure 1E). This indicates that WIP1 negatively regulated p53 in vivo, and p53 activation, in turn, resulted in faithful upregulation of the reporter gene. Further analysis of $p 21$-EGFPpositive cells in the SVZ revealed that they coexpressed NESTIN 
A

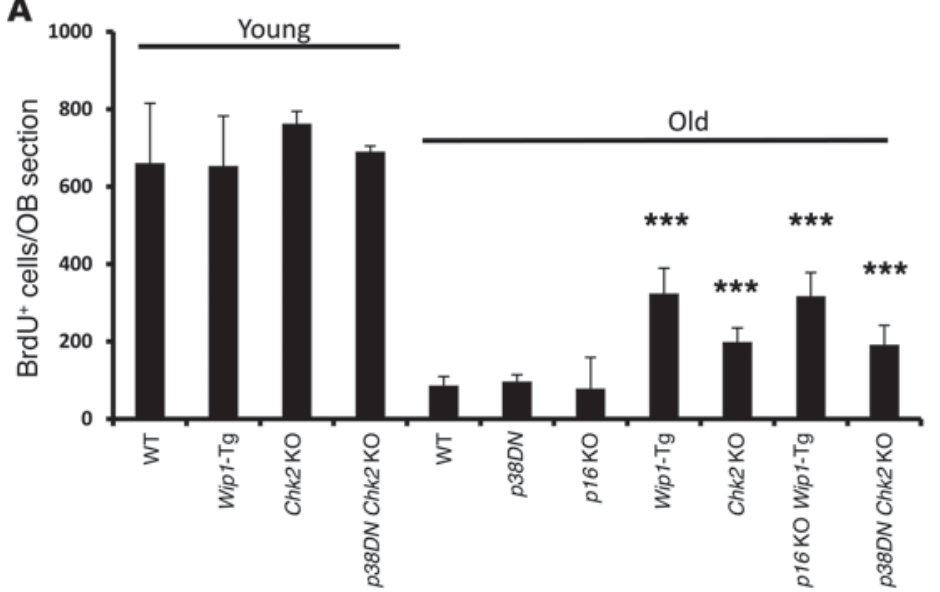

D

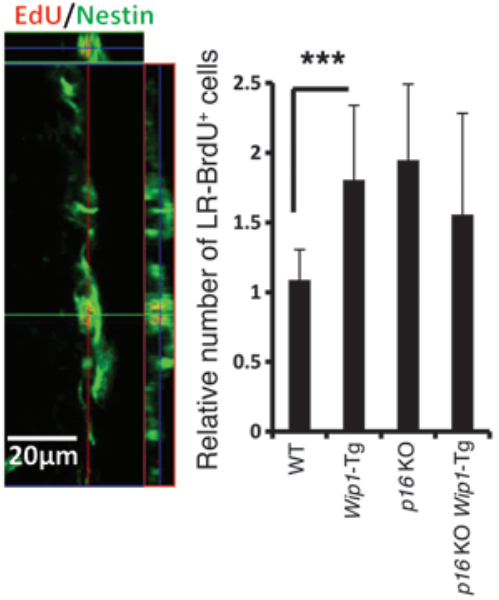

B EdU/NEUN
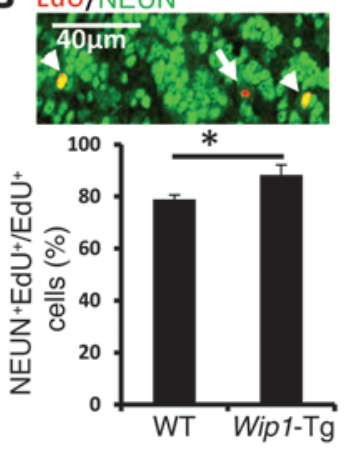

$\mathbf{E}$

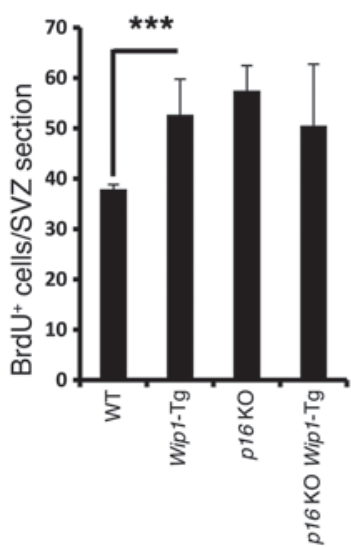

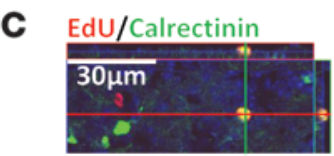

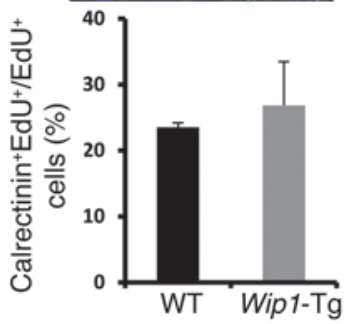

$\mathbf{F}$

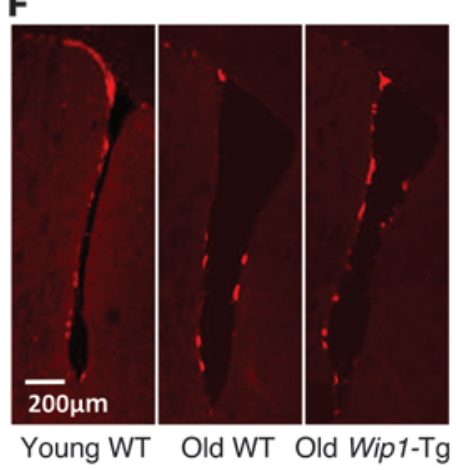

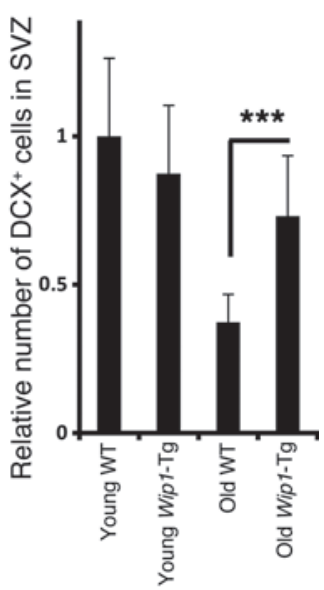

Figure 2

Wip1-Tg expression improves new neuron formation and NSC activity in aged mice. (A) Quantification of BrdU-positive cells in OBs of young (3- to 4-month-old) and old (16-to 18-month-old) mice of different genotypes. Data are mean \pm SEM. (B) Quantification of NEUN-positive cells over total newly formed cells, and a representative photograph of EdU/NEUN colabeling 1 month after EdU injection in aged Wip 1-Tg mice. Arrowheads indicate examples of EdU-positive nuclei that colocalize with NEUN. A arrow indicates an EdU-positive, NEUN-negative cell. Data are mean \pm SEM. (C) Quantification of the proportion of calretinin-positive newborn neurons (calretinin+EdU ${ }^{+}$cells) over total EdU-positive cells (EdU+ cells), and a representative photograph of EdU/calretinin colabeling 1 month after EdU injection in aged Wip1-Tg mice. Data are mean \pm SEM. (D) Representative image of EdU/NESTIN colabeling from aged Wip 1-Tg mice and quantification of LR-BrdU-positive cells per SVZ section

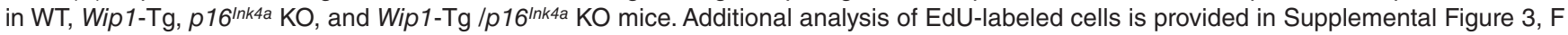
and G. Data are mean \pm SEM. (E) Quantification of short-term incorporation of BrdU, a proliferation marker of NPCs. Data are mean \pm SEM. (F) Quantification of DCX-positive cells in young and aged WT and Wip1-Tg SVZs. Representative photographs of DCX staining in the SVZ areas of young and aged WT and aged Wip1-Tg mice, and quantification of DCX-positive area in the SVZ. Data are mean \pm SD. ${ }^{\star} P<0.05 ;{ }^{* \star *} P<0.005$. Scale bar: $40 \mu \mathrm{m}$ (B); $30 \mu \mathrm{m}$ (C); $20 \mu \mathrm{m}$ (D); $200 \mu \mathrm{m}$ (F).

and SOX2 but were negative for neuroblast markers (Supplemental Figure 1F). We further found an age-dependent increase in $p 21$ EGFP signal in $\mathrm{LeX}^{+} \mathrm{CD} 24-$ cells. This increase was significantly attenuated in Wip1-Tg mice (Figure 1E and Supplemental Figure $1 \mathrm{G})$; Wip1 was only moderately overexpressed in this transgene (Supplemental Figure 1H). Importantly, FACS-sorted p21-EGFPpositive cells coexpressed NESTIN and SOX2 and formed secondary NSPs (Supplemental Figure 1I), which confirmed that the p53 activity was indicative of NPCs. However, the number and size of NSPs derived from $p 21$-EGFP-positive cells was markedly reduced compared with NSPs from EGFP-negative cells (Supplemental Figure 1I). Interestingly, p21-EGFP-positive cells also produced fewer DCX-positive neuroblasts (Figure 1F), suggesting that p53 might be involved in regulating the neurogenic potential of NSCs.
Next, we established NSPs from 2- and 9-month-old mice and analyzed EGFP expression in the NSPs. NSPs from older (9-month-old) WT mice as well as young Wip1-deficient mice contained more EGFP-positive cells than those from young (2-monthold) WT mice (Figure 1, G and H). Analysis of active p53, based on phosphorylation of serine 23 (human serine 20), consistently showed more positive staining in the SVZs of old mice compared with those of young mice (Supplemental Figure 1H). This confirmed that p53 activity is upregulated in the SVZ during aging.

Activation of $\mathrm{p} 53$ is sufficient to suppress neuron production (10). It is not known, however, whether specific repression of p53 activity in NPCs may be useful for improving new mature neuron formation in aged mice. To test whether suppression of p53 is sufficient for increasing neuron production in vivo, we analyzed 

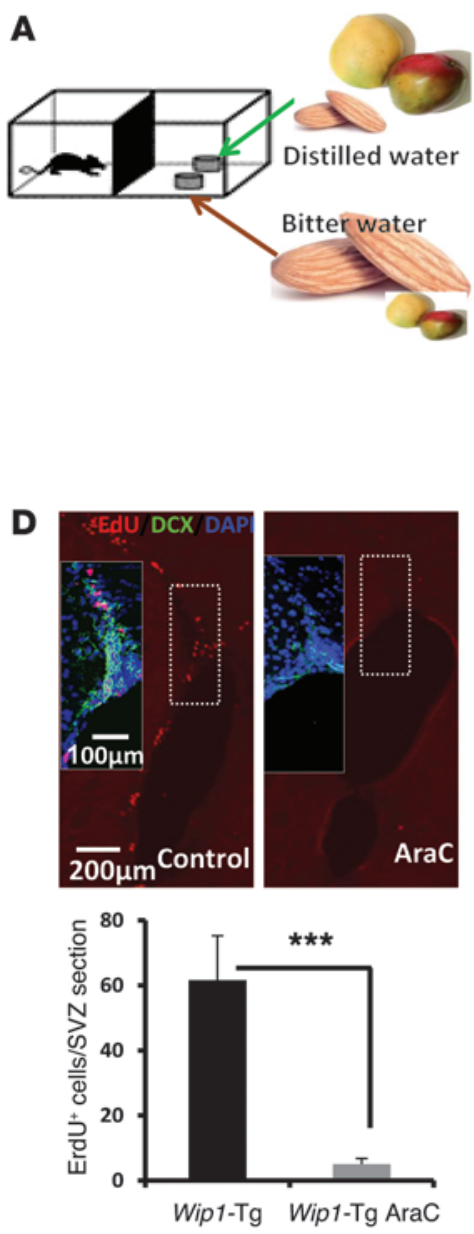
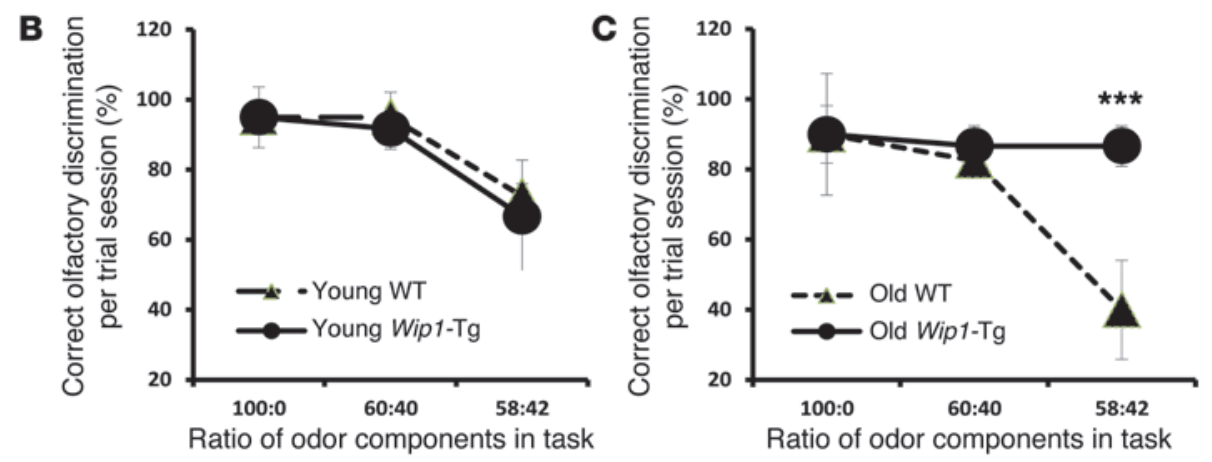
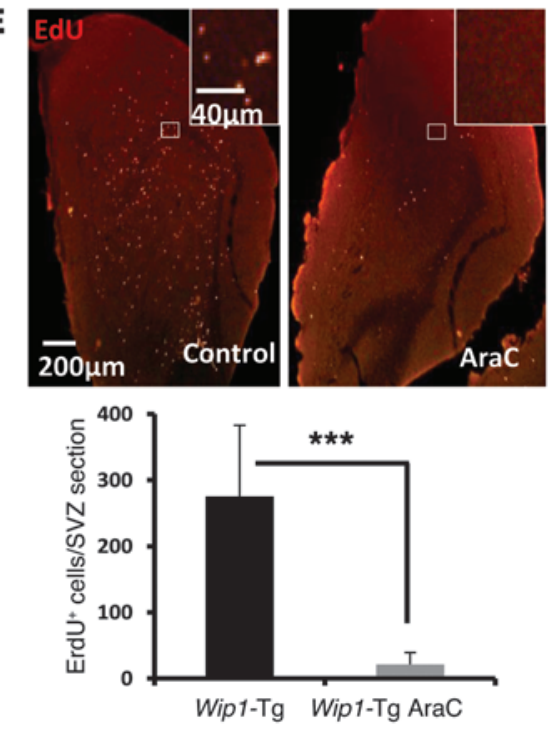

$\mathbf{F}$

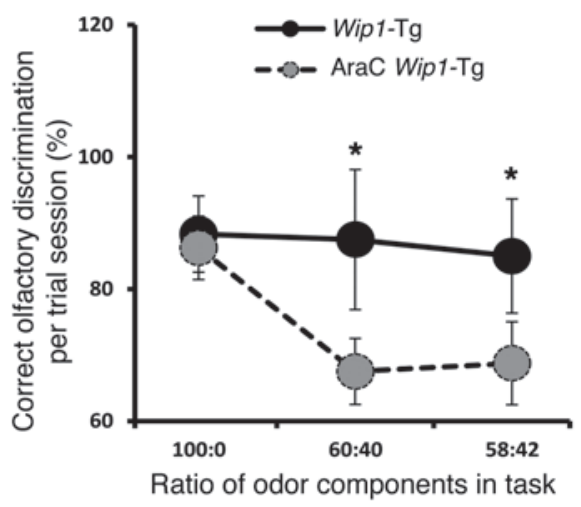

Figure 3

Wip1-Tg mice exhibit improved fine odor discrimination. (A) The design of the fine odor discrimination test. Mice were deprived of water for 2 days and trained to identify distilled water. The distilled water was coupled with an odor mixture of mango and almond; however, mango was the main component. In another dish, bitter water was coupled with a mixture in which almond was the main component. Different proportions of the odors were used for the performance test. (B) Test performance of young (3- to 4-month-old) WT and Wip1-Tg mice shows no differences in fine odor discrimination. Data are mean \pm SEM. (C) Test performance of old (16- to 18-month-old) WT and Wip1-Tg mice shows a significantly better fine odor discrimination of Wip1-Tg. Data are mean \pm SEM. (D) Representative images and quantification of EdU labeling (2-hour pulse) of neural progenitors. Mice were treated with AraC for 1 month (described in Methods). Insets show colabeling of EdU, DCX, and DAPI. Note a decrease in both EdU- and DCX-positive cells in AraC-treated mice. Data are mean \pm SEM. (E) Representative images and quantification of EdU-positive cells 1 month after EdU labeling in control mice and in mice treated with AraC. AraC treatment started right before EdU injection. Data are mean \pm SEM. (F) Test performance of AraC-treated and control 1-year-old Wip1-Tg mice. Note that there is a significant reduction in performance after AraC treatment. Data are mean \pm SEM. ${ }^{*} P<0.05 ;{ }^{* * *} P<0.005$. Scale bar: $200 \mu \mathrm{m}$ (D and E); $100 \mu \mathrm{m}$ (D, inset); $40 \mu \mathrm{m}$ (E, inset).

neuron formation in $p 53 \mathrm{KO}$ mice. Our analysis showed that new neuron formation in the $\mathrm{OB}$ was significantly greater in $p 53 \mathrm{KO}$ mice than in WT mice (Supplemental Figure 2A), suggesting that suppressing p53 activity could potentially ameliorate the aginginduced changes observed in the OB of old mice.

Wip1 overexpression increases neuron formation. Multiple factors are involved in the aging process, making it unlikely that a single molecule can fully rescue a decline in neuron production in the OB (1). To systematically determine the best possible combination of factors for improving neuron production, we analyzed mice with genetic backgrounds that have been implicated previously in aging. Specifically, we wanted to address the complementary role of WIP1 in potential modulation of the p53 pathway as well as to investigate other mechanisms. For that, we used mice of different genetic backgrounds, including a Wip1 transgenic line (Wip1-Tg) that expresses Wip1 under the control of a ubiquitin C promoter (11). This transgenic model produces a physiological 3.5- to 5-fold upregulation of Wip1 mRNA in the SVZ (Supplemental Figure 1H). A KO of $p 16^{\text {Ink } 4 a}$ was shown previously to rescue NSC numbers during aging but had only a marginal effect on neuron production in the OB (1). Thus, we crossed Wip1-Tg mice with $p 16^{\text {Ink4a }} \mathrm{KO}$ mice to look for potential synergistic effects. Our previous analysis showed that $\mathrm{p} 38^{\mathrm{MAPK}}$ is involved in controlling the aging-induced decline in $\beta$ cell proliferation (11). Therefore, we used mice that expressed a dominant-negative form of $\mathrm{p} 38^{\mathrm{MAPK}}$ ( $\mathrm{p} 38 \mathrm{DN}$ ) to investigate the role of this pathway in brain function. Finally, our analysis showed that Chk2, a checkpoint protein and a dephosphorylation target of WIP1 that is an upstream kinase of p53 (12), might play a role in some Wip1 KO mouse phenotypes, including regulation of NSP size and number in vitro and proliferation of progenitors in vivo 
A

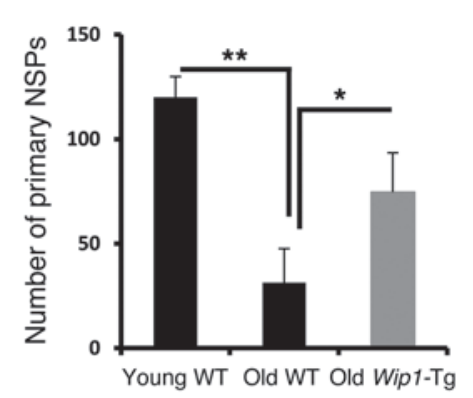

C
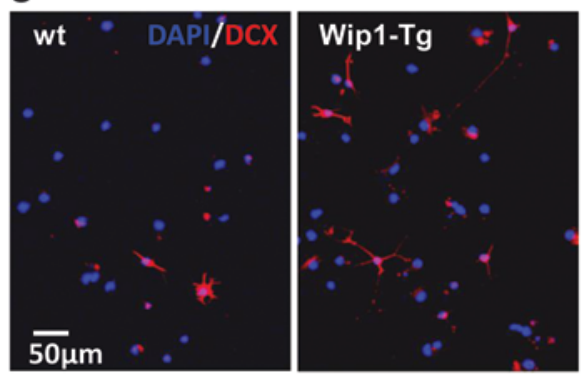

B
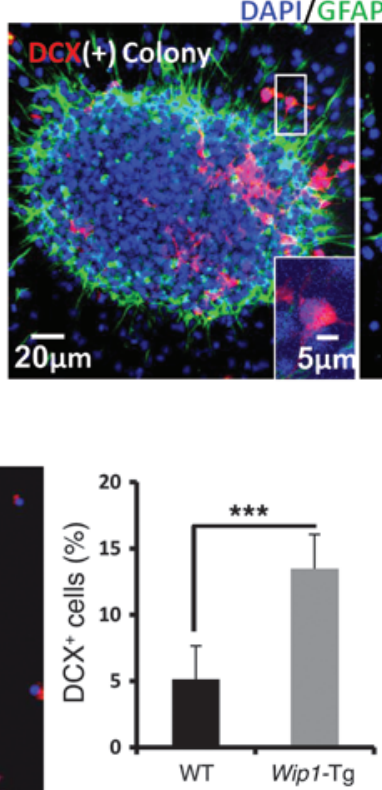

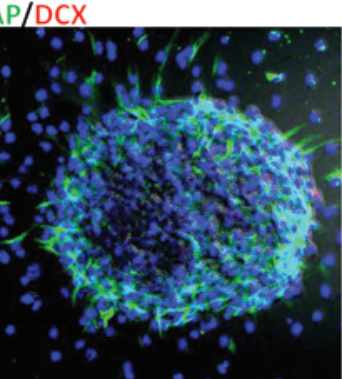

D

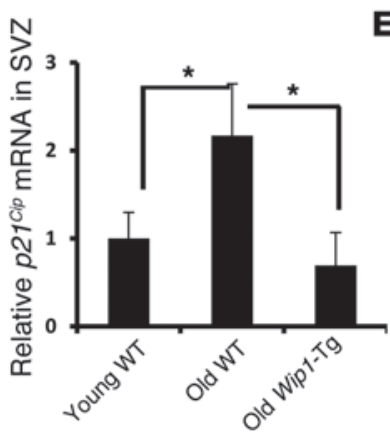

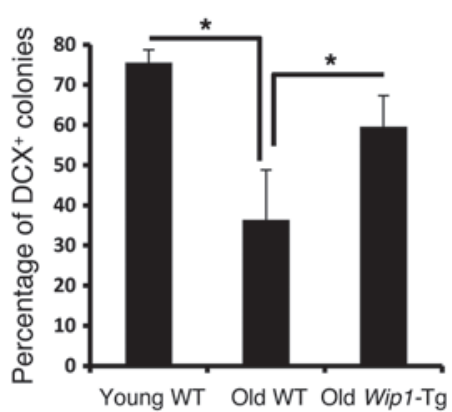

E

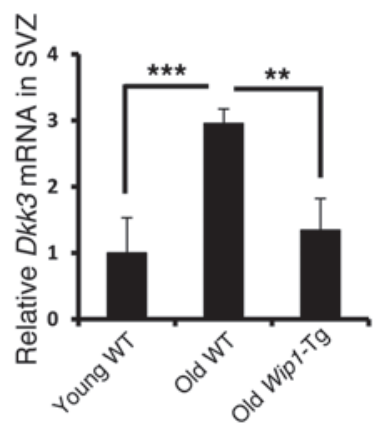

$\mathbf{F}$

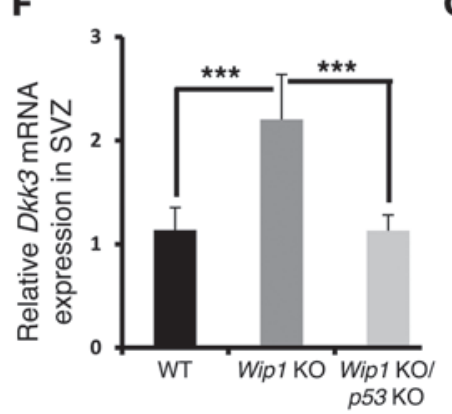

G $\quad$ m21-EGFP-

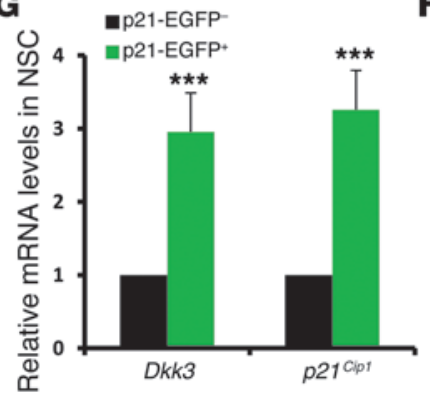

H

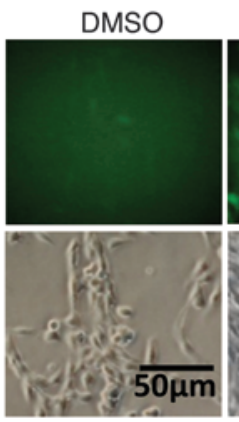

Nutlin

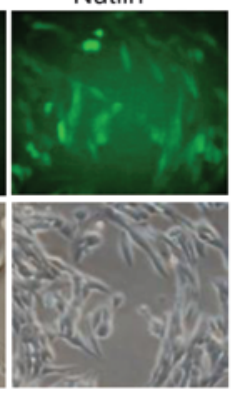

I

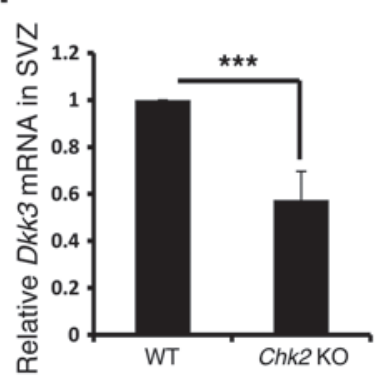

Figure 4

In vitro characterizations of NSCs from Wip1-Tg mice. (A) Quantification of total numbers of primary NSPs derived from young (3- to 4-month-old) and old (16- to 18-month-old) WT mice and old Wip1-Tg mice. Data are mean \pm SD. (B) Sample images of 2 NSP-formed colonies with (left) or without (right) DCX-positive neuroblasts (red cells). The colonies underwent 2 days of differentiation. Quantification of NSP-formed colonies with DCX-positive neuroblasts in young and old WT mice as well as in old Wip1-Tg mice. Data are mean \pm SEM. (C) Representative images of DCX staining after 2 days of differentiation of old WT and Wip1-Tg primary NPCs. Data are mean \pm SD. (D) Real-time PCR analysis of $p 21$ Cip expression in the SVZs in young and old WT mice as well as in old Wip1-Tg mice. Data are mean \pm SEM. (E) Real-time PCR analysis of Dkk3 mRNA expression in the SVZs in young and old WT mice as well as in old Wip1-Tg mice. Data are mean \pm SEM. (F) Real-time PCR analysis of Dkk3 mRNA expression in the SVZs of young WT, Wip $1 \mathrm{KO}$, and Wip1 KO/p53 KO mice. Data are mean \pm SEM. (G) Real-time PCR analysis of relative expression of $D k k 3$ and $p 21^{\text {Cip } 1 ~ i n ~ s o r t e d ~ p 21-E G F P-p o s i t i v e ~ a n d-n e g a t i v e ~ N S C s . ~ D a t a ~ a r e ~ m e a n ~} \pm$ SEM. (H) Representative images of DKK3GFP-positive cells with DMSO and Nutlin treatment. (I) Analysis of $D k k 3$ mRNA expression in freshly isolated cells from SVZ areas of 1-year-old WT and Chk2 KO mice. Data are mean \pm SEM. ${ }^{*} P<0.05 ;{ }^{* \star} P<0.01 ;{ }^{* \star} P<0.005$. Scale bar: $20 \mu \mathrm{m}(\mathbf{B}) ; 5 \mu \mathrm{m}(\mathbf{B}$, inset); $50 \mu \mathrm{m}(\mathbf{C}$ and $\mathbf{H})$.

(Supplemental Figure 2, B-D). These observations prompted us to include Chk2 $\mathrm{KO}$ mice in the analysis as well.

To assess adult neurogenesis in these strains of mice (Wip1-Tg, $p 16^{\text {Ink4a }} \mathrm{KO}$, Wip1-Tg/p16 $6^{\text {Ink4a }} \mathrm{KO}, p 38 D N$, Chk2 KO, and WT mice), we injected BrdU into mice aged 3-4 months (young mice) or aged 16-18 months (old mice) and analyzed BrdU-positive neurons in the OB 1 month later (Figure 2A). At this stage, most BrdU-positive cells expressed the neuronal marker NEUN (Figure 2B) and partially expressed calretinin, a marker of mature GABAergic neurons (Figure 2C), so we counted these cells to determine the efficiency of adult neurogenesis in different genetic backgrounds according to mouse age. We found no differences in young mice of differ- ent genetic backgrounds, but some genetically modified aged mice showed a significant improvement in neuron formation compared with aged WT mice (Figure 2A). Specifically, aged Wip1-Tg and Chk2 $\mathrm{KO}$ mice displayed a marked rescue of the aging-induced decline in adult neurogenesis in the OB (Figure 2A). Importantly, newly born cells analyzed in the OBs of aged Wip1-Tg and Chk2 KO mice were mainly neurons (Figure 2, B and C, and Supplemental Figure 3, A and B). Taken together, these data suggest that Wip1 overexpression substantially improve adult neurogenesis in the OBs of aged mice.

We did not observe a significant increase in the number of BrdU-positive cells in the OBs of $p 16^{\text {Ink4a }} \mathrm{KO}$ mice or $p 38 D N$ mice, suggesting that attenuation of $\mathrm{p}^{38^{\mathrm{MAPK}}}$ or $\mathrm{p} 16^{\mathrm{Ink} 4 \mathrm{a}}$ is not 
A

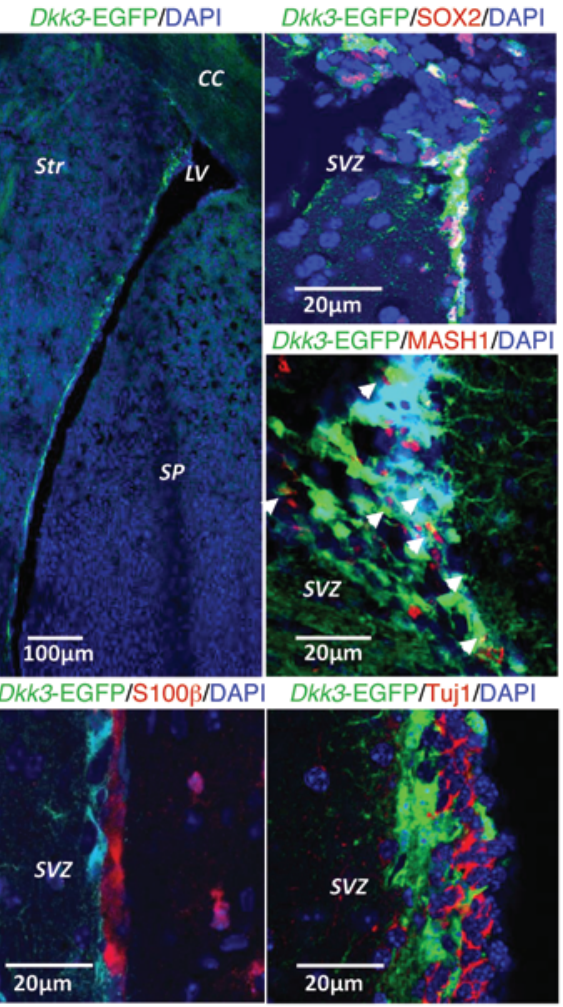

B

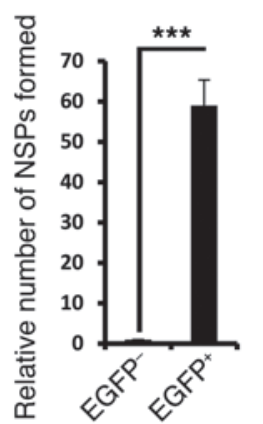

C
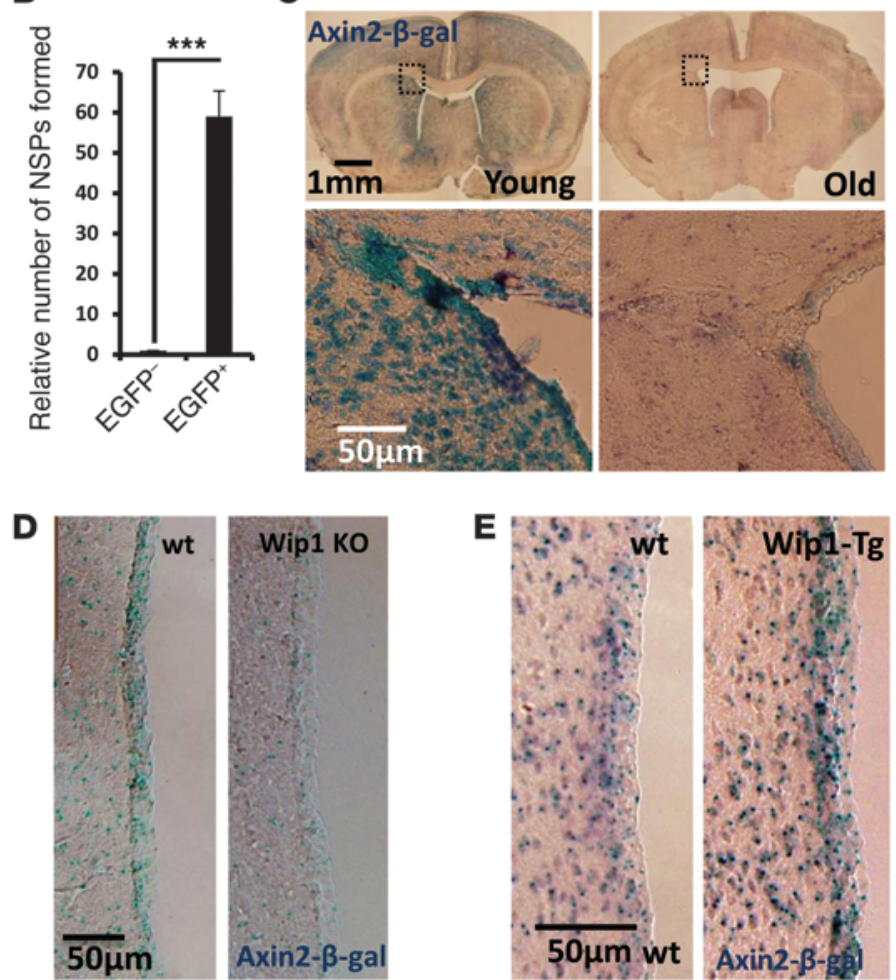
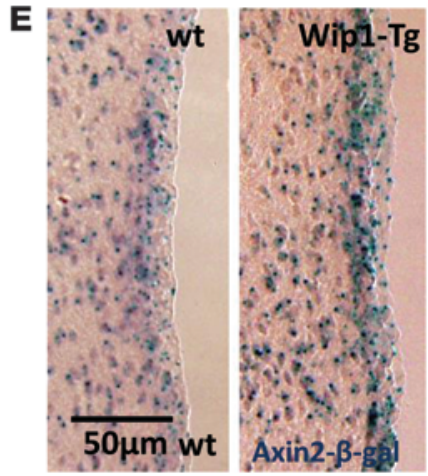

$\mathbf{F}$

G
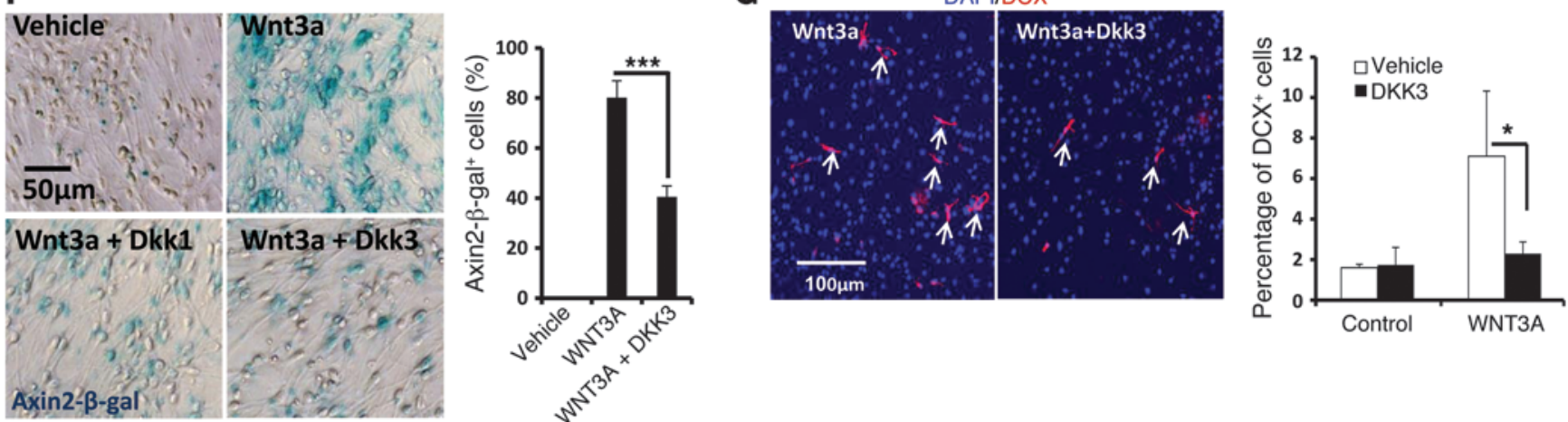

Figure 5

Expression and functional analysis of DKK3. (A) Representative images of EGFP (green) expression in the brains of Dkk3-EGFP-Tg mice. An overview of the lateral ventricle area is shown. These Dkk3-EGFP-positive cells are largely positive for SOX2, partially positive for MASH1 (highlighted with white arrowheads), and negative for $S 100 \beta$ and Tuj1. (B) Quantification of relative numbers of NSPs formed from total Dkk3EGFP-positive and -negative cells SVZ cells. Detailed analysis of NSP formation is presented in Supplemental Figure 4E. Data are mean \pm SD. (C) Representative images of Axin2- $\beta$-gal staining in young (2-month-old) and old (2-year-old) forebrains. Enlarged images are shown below. (D) Representative images for Axin2- $\beta$-gal activity in SVZs of 4-month-old WT and Wip1 KO mice. (E) Representative images for Axin2- $\beta$-gal activity in SVZs of 1-year-old WT and Wip1-Tg mice. (F) Representative images of Axin2- $\beta$-gal staining of cultured NPCs after 2 days treatment with vehicle, WNT3a, WNT3a plus DKK3, or WNT3a plus DKK1. Quantification of Axin2- $\beta$-gal-positive cells in different groups. Data are mean \pm SEM. (G) Analysis of neuroblasts formation 2 days after differentiation of NPCs on coverslips in the presence of WNT3a or WNT3a and DKK3. Representative images of DCX staining (arrows indicate neuroblasts). Quantification of the effects of WNT3 and DKK3 on formation of DCX-positive neuroblasts. Data are mean \pm SD. ${ }^{\star} P<0.05$; ${ }^{\star \star \star} P<0.005$. Scale bar: $100 \mu \mathrm{m}$ (A, top left, and $\left.\mathbf{G}\right) ; 20 \mu \mathrm{m}$ (A, top right, middle, and bottom); $1 \mathrm{~mm}$ (C, top row); $50 \mu \mathrm{m}$ (C, bottom row, and $\mathbf{D}-\mathbf{F})$.

sufficient to override aging-induced changes in the OB. Wip1$\mathrm{Tg} / \mathrm{p} 16^{\text {Ink4a }} \mathrm{KO}$ mice consistently showed no additional improvement in new neuron formation over that seen in Wip1-Tg mice, while Chk2 $\mathrm{KO} / p 38 D N$ mice had an improvement similar to that of Chk2 KO mice (Figure 2A). We further found that Wip1 overexpression did not change the morphology of new neurons
(Supplemental Figure 3C). We also observed no difference in apoptosis of neuroblasts (Supplemental Figure 3D) and cell cycle reentry of NSCs (Supplemental Figure 3E).

To investigate whether the number of newly formed neurons in the $\mathrm{OB}$ was directly (i.e., linearly) related to the number of NSCs and progenitors, we next analyzed BrdU label-retaining 
A

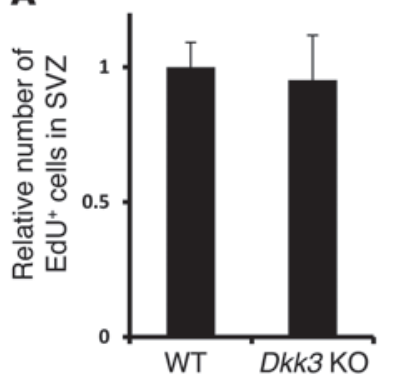

D
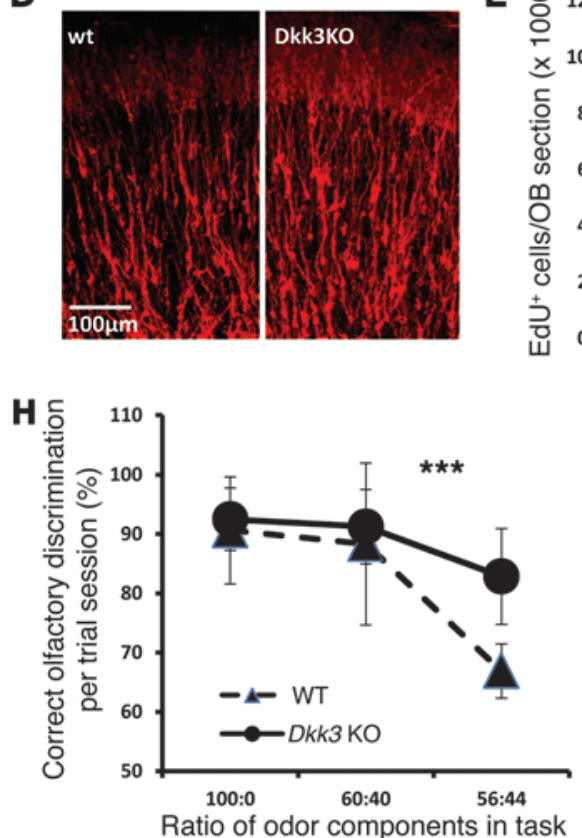

B

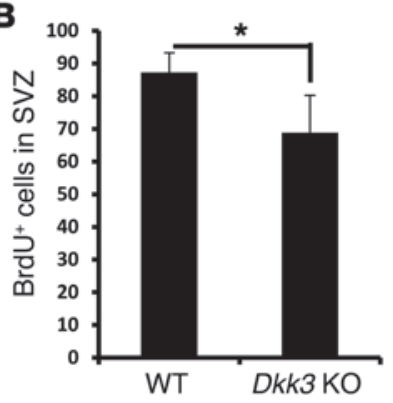

E

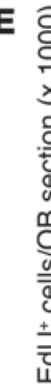

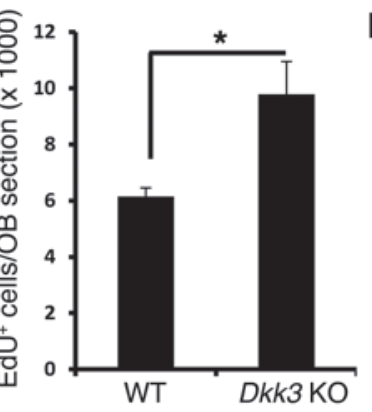

C

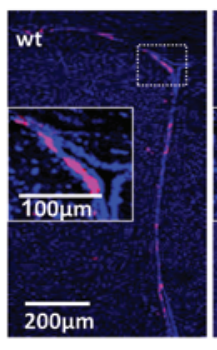

$\mathbf{F}$

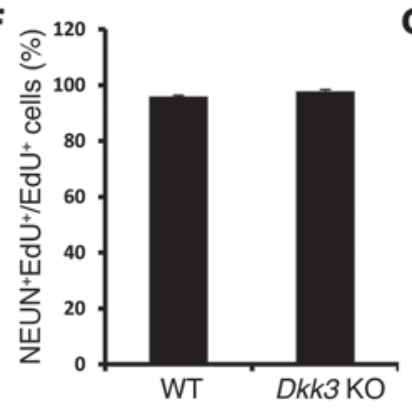

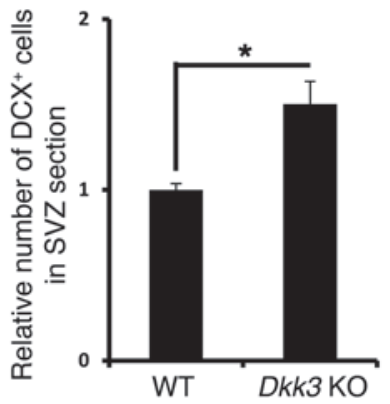

G

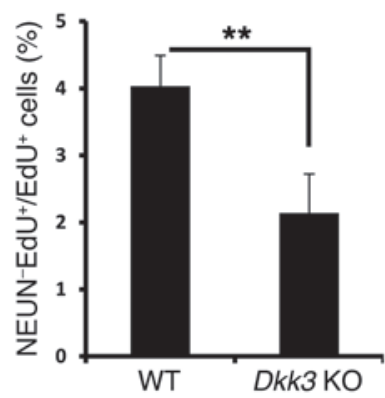

I

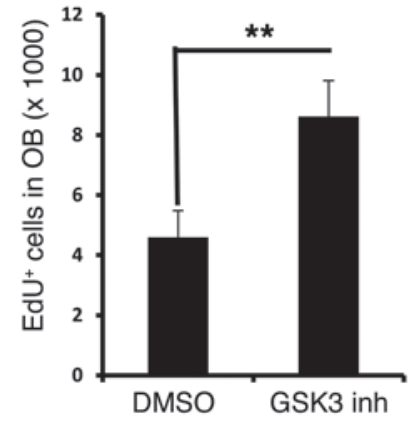

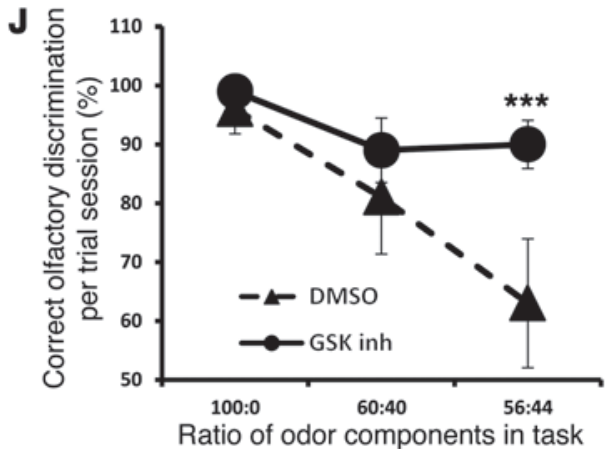

\section{Figure 6}

Characterization of adult neurogenesis in Dkk3 KO mice. (A) Quantification of long-term EdU-retaining cells in SVZs of 6-month-old WT and $D k k 3$ KO mice. Data are mean \pm SEM. (B) Quantification of labeled cells after a 2-hour BrdU pulse of same set of mice as in A. Data are mean \pm SEM. (C) Analysis of DCX-positive cells in SVZs of 6-month-old WT and Dkk3 KO mice. Data are mean \pm SEM. (D) Photographs showing DCX-positive cells in the OBs of WT and Dkk3 KO mice. (E) Quantification of EdU-positive cells that have integrated in the OBs 1 month after EdU labeling. Data are mean \pm SEM. (F) Quantification of NEUN-positive cells over total EdU-positive cells in WT and Dkk3 KO mice. Data are mean \pm SEM. (G) Quantification of the proportion of NEUN-negative newborn cells over total EdU-positive cells in WT and Dkk3 KO mice. Data are mean \pm SEM. (H) Test performance of WT and Dkk3 KO mice at 3 to 5 months of age. Data are mean \pm SEM. (I) Quantification of new cell (EdU) formation in 14-month-old mice after treatment with a GSK3 inhibitor (SB216763). Data are mean \pm SEM. (J) Test performance of WT mice at 8 months of age 4 weeks after injection of vehicle or a GSK3 inhibitor (SB216763). Data are mean \pm SEM. ${ }^{*} P<0.05 ;{ }^{* *} P<0.01$; ${ }^{* \star *} P<0.005$. Scale bar: $200 \mu \mathrm{m}(\mathbf{C}) ; 100 \mu \mathrm{m}(\mathbf{C}$, inset, and D).

cells in the SVZ. Specifically, we conducted long-term labelretaining experiments to analyze quiescent NSCs and short-term BrdU labeling to analyze progenitors. We found that Wip1-Tg and $p 16^{\text {Ink4a }} \mathrm{KO}$ mice had similar rescue phenotypes, in terms of the number of long-term label-retaining NSCs (Figure 2D and Supplemental Figure 3, F and G) and progenitor proliferation (Figure 2E). Consistently, the number of neuroblasts in the SVZ regions of aged mice was rescued in Wip1-Tg mice (Figure $2 \mathrm{~F}$ and Supplemental Figure $3 \mathrm{H})$. As $p 16^{\text {Ink } 4 a}$ deficiency had only a marginal effect on improvement of neurogenesis in the OB (Figure $2 \mathrm{~A})$, this suggests that rescue of NSC self-renewal alone is not sufficient for reversing a decline in the formation of neurons during aging. In turn, this implies that other mechanisms may be equally important for rescuing neuron formation in aging mice and that some of these mechanisms might depend on the regulation of neuronal differentiation by $\mathrm{p} 53$ and WIP1.

Wip1 overexpression rescues an age-related decline in olfaction. Agerelated decrease in neuron formation has been proposed as one cause of brain function decline (13). However, evidence has been scarce regarding whether a rescue of neuron production can actually improve brain function. To look at brain functions related to the OB, we used a behavioral olfaction test that has been shown to correlate with age-related decline in new neuron formation (13). In this test, mice were trained to distinguish between mixtures containing different fractions of mango and almond flavors, which have distinct aromas (Figure 3A). The mango and almond 
flavors were mixed with either distilled or bitter water when mango or almond flavor, respectively, was the major component of the mixture. For mixtures containing more than $60 \%$ of either substance, the success rate in discriminating between the different smells was comparable in WT and Wip1-Tg mice (Figure 3, B and $\mathrm{C}$ ). This indicates that mice with these genotypes were not defective in memorizing these smells during the test phases. However, when each mixture contained only $58 \%$ of either almond or mango flavor, aged Wip1-Tg mice performed significantly better than aged WT mice (Figure $3 \mathrm{C}$ ). There was no difference in performance between young WT and young Wip1-Tg mice (Figure $3 \mathrm{~B}$ ), indicating that overexpressing Wip1 specifically improved olfactory function in aged mice.

Next, we tried to understand whether new neuron formation was responsible for increased olfactory function in Wip1-Tg mice. To uncouple the role of Wip1 overexpression in NPCs from potential systematic effects, we carried out cytosine arabinoside (AraC) infusion experiments to specifically eliminate neural progenitors and neuroblasts in the SVZ (Figure 3D). The AraC treatment also efficiently suppressed new neuron production in the OB (Figure 3E). Our analysis showed that AraC-induced arrest of neurogenesis for 1 month was sufficient to significantly diminish the performance of Wip1-Tg mice in fine odor discrimination tests (Figure 3F). These data support the notion that changes in olfactory function with age could reflect changes in neuron production in the $\mathrm{OB}$, which in turn could be controlled by WIP1.

Wip1 overexpression rescues age-related NSP deficit. To dissect the mechanism underlying improved neuron formation in the OBs of aged Wip1-Tg mice, we first performed in vitro experiments. Specifically, we isolated SVZ cells from young and aged WT and Wip1-Tg mice and established NSP cultures. There were fewer NSPs from aged mice than from young animals, and NSPs from aged mice were smaller (Figure 4A and Supplemental Figure 2I). Consistent with our in vivo data showing that aged Wip1-Tg mice have higher NSC activity (Figure 2, D-F), the number of primary NSPs established from aged Wip1-Tg mice was significantly higher than the number established from aged WT mice (Figure 4A). This difference in the number of primary NSPs was age specific, as we did not observe a difference between young WT and Wip1-Tg mice (Supplemental Figure 2J). Furthermore, the size of the NSPs did not differ between WT and Wip1-Tg mice (Supplemental Figure 2K), suggesting that WIP1 primarily regulates NSP number and thus regulates NSC selfrenewal but not the proliferation of more differentiated progenitors.

Next, we analyzed neuronal differentiation using NSPs. Two days after withdrawal of FGF and EGF, the majority of NSPs from young mice became attached to the glass slide and some of DCXpositive neuroblasts began to migrate out. The fraction of NSPs that generated DCX-positive neuroblasts was significantly higher in young mice, suggesting that neuronal differentiation is impaired in aged mice. Importantly, NSPs from aged Wip1-Tg mice showed improved neurogenic capacity (Figure 4B). Consistently, differentiation of single cells from primary NSPs showed an increase in neuronal commitment in Wip1-Tg mice (Figure 4C), indicating that WIP1 regulates the neuronal differentiation of NSCs. As the data in $p 16^{\text {Ink } 4 a} \mathrm{KO}$ mice show that regulation of NSC numbers was not sufficient to ameliorate a decline in neuron formation in the OBs of aged mice (Figure 2A), subsequent analysis focused on the mechanisms of WIP1-dependent regulation of neuronal differentiation.

WIP1 controls the expression of DKK3, a WNT antagonist. To better understand the potential mechanisms of WIP1-dependent reg- ulation of neuronal differentiation, we performed preliminary microarray analysis to identify differences in gene expression profiles in NSPs from aged WT mice and aged Wip1-Tg mice. WNT signaling was implicated previously in neuronal fate determination (14), and we found that several WNT-related genes, including a gene encoding a putative WNT signaling inhibitor, Dkk3, was downregulated 2.2-fold in NSPs from aged Wip1-Tg mice. To verify this, we performed RT-PCR analysis and confirmed that Dkk3 mRNA levels were significantly increased in aged WT mice but not in aged Wip1-Tg mice, similar to p21 Cip mRNA levels (Figure 4, D and E). Importantly, a similar increase in Dkk3 expression was found in the SVZs of Wip1 KO mice (Figure 4F). Recent studies highlighted the importance of DKK1 in the hippocampus (4); however, the level of Dkk1 mRNA was not regulated in a Wip1-dependent manner or with aging in the SVZs (Supplemental Figure 4, A and B).

A recent study showed that Dkk3 mRNA expression could be regulated in a p53-dependent manner (15). To verify this, we first analyzed mice that are deficient in Wip1 and $p 53$ and found that removal of $p 53$ was sufficient to reduce the level of Dkk3 mRNA (Figure 4F). This suggests that WIP1 controls DKK3 through p53. To further validate the role of p53-dependent regulation of DKK3 in a WT context, we sorted EGFP-positive and -negative cells from a pool of primary NSPs derived from the $\mathrm{p} 53$ reporter mice, $p 21$-EGFP transgenics. RT-PCR analysis showed that Dkk3 mRNA expression was significantly higher in $p 21$-EGFP-positive cells (Figure 4G). Importantly, Nutlin treatment that specifically activates p53 efficiently induced Dkk3 reporter activity (Figure $4 \mathrm{H}$ ), suggesting a strong correlation between DKK3 expression and p53 activity. Consistently, knocking out Chk2, an upstream kinase that activates p53 in NPCs, reduced Dkk3 mRNA expression in SVZ cells of aged mice (Figure 4I). Taken together, our data indicate that WIP1 regulates p53-dependent DKK3 expression.

DKK3 is expressed in SVZ NSCs. To understand the cell specificity of DKK3 expression, we used a transgenic mouse strain that carries an EGFP reporter under the control of a Dkk3 promoter (16). First, we confirmed DKK3 expression in the CA3 region of the hippocampus (17). Next, we analyzed expression in the SGZ and SVZ, 2 neurogenic zones in the adult mouse brain. We found no Dkk3-EGFP expression in the SGZ. In contrast, Dkk3-EGFP expression was high in the SVZ (Figure 5A and Supplemental Figure 4C). Colocalization analysis confirmed that the majority of Dkk3-EGFP-positive cells coexpressed SOX2, and some were also positive for MASH1 but negative for TUJ1/DCX and S100 $\beta$ expression (Figure 5A), suggesting that DKK3 expression is restricted to the early stages of neurogenesis. To confirm this, we analyzed the ability of DKK3-expressing cells to form NSPs. We found that only FACS-sorted Dkk3-EGFP-positive cells were able to form NSPs (Figure 5B and Supplemental Figure 4D); this was in sharp contrast to the nearly complete lack of NSPs from Dkk3-EGFP-negative cells. These data strongly argue that DKK3 expression is restricted to the early stages of neurogenesis, and the expression pattern of DKK3 suggests that it may regulate NPCs in an autocrine and/or paracrine manner.

DKK3 inbibits canonical WNT signaling and neurogenesis. DKK3 is a putative WNT inhibitor, and WNT signaling has been implicated in neuronal fate determination (14). Thus, our data may suggest that WIP1 regulates neurogenesis during aging via DKK3-dependent regulation of WNT. This was supported by the observation that Dkk3 mRNA expression was higher in aged mice than in young mice (Figure 4E). Furthermore, in vivo analysis of mice 
with the Axin2- $\beta$-gal reporter gene, which is a reporter of WNT signaling (18), showed age-dependent downregulation of WNT signaling in the SVZ region (Figure $5 \mathrm{C}$ ). Consistent with WIP1and p53-dependent regulation of DKK3 and WNT signaling, we observed an upregulation of Dkk3-EGFP signal (Supplemental Figure 4E) and a downregulation of Axin2- $\beta$-gal reporter activity in Wip1-deficient mice (Figure 5D) and an upregulation of Axin2$\beta$-gal reporter activity in Wip1-Tg mice (Figure 5E).

DKK3 has been implicated in the regulation of both canonical and noncanonical WNT pathways (19). To determine the impact of DKK3 specifically on canonical WNT signaling, as it plays an important role in adult neurogenesis, we analyzed the effect of DKK3 on NSPs derived from Axin2- $\beta$-gal reporter mice. Consistent with a previous report (20), we found that the NSPs had very low basal WNT activity that was efficiently upregulated in the presence of WNT3a, as determined by $\beta$-gal staining (Figure $5 \mathrm{~F}$ ). Importantly, inclusion of DKK3 significantly diminished WNT3ainduced Axin2- $\beta$-gal activity to an extent similar to that induced by DKK1, a classical WNT inhibitor (Figure 5F). Taken together, our data suggest that DKK3 could behave as an inhibitor of canonical WNT signaling in NSCs and progenitors.

As canonical WNT signaling has been shown to play an important role in fate commitment toward neuronal lineages (14), we next tested the effect of DKK3 on neuronal differentiation. The presence of WNT3a significantly increased the number of DCXpositive neuroblasts, while there was no increase in DCX-positive neuroblasts when DKK3 was present (Figure 5G). This indicates that DKK3 upregulation, including upregulation during aging, could inhibit WNT-induced neurogenesis.

$D K K 3$ inhibits neuronal commitment with aging. In order to investigate the function of DKK3 in adult neurogenesis in vivo, we next turned to the analysis of 6-month-old Dkk3 KO mice. At this age, there is a significant reduction in neurogenesis, thus allowing us to understand the involvement of different pathways in aging. Analysis of 5-ethynyl-2'-deoxyuridine (EdU) label-retaining cells showed that a deficiency of $D k k 3$ did not affect the number of quiescent NSCs in SVZ region (Figure 6A). After a 2-hour BrdU pulse, a proliferation assay showed that DKK3 had only a marginal role in regulating proliferation of NPCs (Figure 6B). The minor reduction in proliferation could be a result of an enhanced neuronal commitment, as the number of DCX-positive neuroblasts was significantly increased in the SVZs of Dkk3 KO mice (Figure 6C). The increased number of neuroblasts was also observed in $\mathrm{OB}$ sections of Dkk3 KO mice, although the morphology was not different from that in WT mice (Figure 6D). Next, we quantified the numbers of newly formed cells in OB generated within 1 month and found a significant increase in Dkk3 KO mice (Figure 6E). The majority of these EdU-positive cells were neurons, as more than $95 \%$ of them were NEUN positive, both in WT and Dkk3 KO mice (Figure 6F). Additionally, the fraction of NEUN-negative cells was reduced in Dkk3 KO mice (Figure 6G), similar to that observed in aged Wip1-Tg mice (Supplemental Figure 3, A and B). Furthermore, the odor discrimination test revealed a significant improvement of olfactory functions in Dkk3 KO mice (Figure 6H). These data strongly support the role of DKK3 in negative regulation of neuronal commitment in vivo.

If an increase in Dkk3 mRNA expression during aging results in suppression of WNT-induced neurogenesis, it is possible that pharmaceutical reactivation of the WNT pathway might improve neuron formation in the OBs of aged mice. To test this possibility, we reactivated WNT signaling (Supplemental Figure 4F) by injecting a GSK3 inhibitor 9 times, once every 2 days. Mice were sacrificed 2 weeks after the last injection. We also included EdU in the first 5 injections to label NSCs/progenitors. Analysis of EdU-positive cells in the OBs revealed that the GSK3 inhibitor improved neurogenesis in the OBs of aged mice (Figure 6I). To investigate whether chemical reactivation of the WNT pathway could result in improvement of olfactory functions, we carried out an odor discrimination test. We found that injections of GSK3 inhibitor were sufficient to significantly improve olfactory functions in mice (Figure 6J), providing strong evidence that some age-related changes are at least partially reversible and can be achieved through pharmacological activation of WNT signaling.

\section{Discussion}

We found previously that Wip1 deficiency attenuated neuron production in the OB via regulation of NSC self-renewal and neuronal differentiation (5). Here, we extended these findings by examining the role of Wip1 in physiologically relevant conditions. We found that Wip1 mRNA was specifically expressed in NSCs and its expression was reduced with aging. Importantly, manipulating WIP1 levels significantly improved adult neurogenesis in the SVZs of aged mice (Figure 1A and Figure 2B). We also found that WIP1 contributes to regulation of p53 during aging, as we observed that many p53-regulated genes were downregulated in NSPs derived from aged Wip1-Tg mice (data not shown). Furthermore, overexpression of Wip1 at physiological levels was sufficient to rescue neuron formation in the OBs and to improve olfaction in aged mice (Figures 2 and 3). These effects of WIP1 were most likely due to its protective effects on both aging-induced decline in NSC self-renewal and on the ability of NSCs to commit to neuronal differentiation, Notably, rescue of NSC self-renewal in the SVZ alone, as seen in $p 16^{\text {Ink } 4 a} \mathrm{KO}$ mice, was not sufficient to improve neurogenesis in the OB (Figure 2, A-E). These data highlight the important role of neuronal differentiation in the regulation of aging-induced decline in adult neurogenesis.

We found that p53 activity increased in NSCs during aging, and p53 is known to control neurogenesis $(5,10)$. However, the molecular mechanisms underlying p53-dependent regulation of neurogenesis during aging are not well characterized. Using $p 53$ reporter mice, we demonstrated that p53 activity is elevated in aged mice. In our working model, upregulation of p53 activity during aging inhibits neuron production in the OB via two complementary mechanisms. First, p53 represses NSC self-renewal by activating cell cycle inhibitors, including $\mathrm{p} 21^{\mathrm{Ci}}$. Importantly, p53 activation during aging does not involve activation of apoptosis-promoting target genes, such as Puma (data not shown), suggesting that p53-dependent regulation on proliferative effects during aging may be uncoupled from p53-dependent apoptosis. A similar effect is achieved through an aging-induced activation of $\mathrm{p} 16^{\mathrm{Ink} 4 a}(1)$. Second, the WIP1/ p53 pathway also suppressed neuronal differentiation through induction of DKK3 (Figures 4 and 5) and subsequent inhibition of WNT signaling, as described herein.

Using a reporter mouse line, we found that the $D k k 3$ promoter was specifically active at early stages of neurogenesis (Figure $5 \mathrm{~A}$ ). This specificity is strongly supported by the fact that only Dkk3EGFP-positive cells were able to form primary neurospheres in vitro (Figure 5B). The lack of DKK3 expression in SGZ region is in 
stark contrast to DKK1 expression, which is expressed primarily in the SGZ and granular neurons and at much lower levels in SVZ region (4). This different expression highlights the regional specificity of mechanisms controlling neurogenesis.

The function of DKK3 in homeostatic tissues is not well understood (21). Encouraged by our in vitro data (Figure 5E), we extended our analysis to Dkk3 KO mice. Strikingly, we found a very specific role of DKK3 in neurogenesis in vivo, namely a regulation of neuronal commitment but not proliferation or self-renewal of NPCs (Figure 6). This function of DKK3 is fully consistent with the role of WNT signaling in neuronal fate commitment $(2,14)$. Indeed, we found that Axin2-LacZ signal induced by WNT3a was strongly inhibited by DKK3 (Figure 5F), suggesting that DKK3 attenuates neurogenesis through blocking WNT signaling.

In contrast to the SGZ $(4,14,22)$, the role of WNT in SVZ neurogenesis is not well characterized. Interestingly, overexpression of TLX, an upstream regulator of canonical WNT signaling, also boosts $\mathrm{OB}$ neuron formation, which was further consistent with our GSK3 inhibitor experiments (Figure 6, I and J). Importantly, injection of a GSK3 inhibitor alone was sufficient to improve neurogenesis and odor discrimination, suggesting a strategy to improve some brain function with aging. In conclusion, we found that WIP1 controls DKK3-dependent neuronal differentiation with aging and propose that pharmacological activation of the WIP1/WNT pathway may have potential to prevent some aspects of functional deterioration of the aging brain.

\section{Methods}

Animals. Wip1 $\mathrm{KO}$ and $p 53 \mathrm{KO}$ (5), Wip1-Tg and p38DN (11), Axin2-lacZ (18), p21-EGFP (9), $p 16^{\text {Ink4a }} \mathrm{KO}$ and Chk2 KO (23), and Dkk3-EGFP-Tg (14) mouse lines have been described previously. The Dkk3 KO mouse line was generated in the lab of Takahisa Furukawa (Institute for Protein Research, Osaka University, Suita, Osaka, Japan), by replacing exons 1-3 with a neomycin cassette, and obtained from RIKEN. Littermates were used for majority of experiments comparing different genotypes, and age-matched litters were used when more than 2 genotypes and/or mice were used and data were combined (Figure 2A, Figure 3, and Supplemental Figure 2, B-D).

Antibodies and reagents. The following primary antibodies were used: $\beta$-gal (MP Biomedicals), NEUN (Chemicon), GFAP (DAKO), Tuj1 (Covance), BrdU (Abcam), phospho-p53 serine 23 (Cell Signaling Technology), DCX (Abcam), NESTIN (BD Pharmingen), SOX2 (Abcam), MASHh1 (BD Pharmingen), DKK3 (Proteintech), LeX antibody (eBioscience), and CD24 (eBioscience). Alexa Fluor 488- or 555-conjugated secondary antibody (Invitrogen) was used. Mouse DKK1, DKK3, and WNT3a proteins (R\&D Systems) were used as well as GSK3 inhibitor SB216763 (Sigma-Aldrich).

$B r d U$ and EdU. To assess activity of NSCs in adult mice, BrdU was injected as described previously described (5) with little modification. In brief, for short-term BrdU injection, intraperitoneal injection of BrdU/EdU (dissolved in $0.06 \mathrm{~N} \mathrm{NaOH}$ in $0.9 \% \mathrm{NaCl}$ and neutralized by HCL before use; $30 \mathrm{mg} / \mathrm{kg}$ body mass) was given 5 times every 2 hours. Mice were sacrificed 2 hours after the last injection. For long-term SVZ BrdU/EdU retention and $\mathrm{OB}$ new neuron formation experiments, $100 \mathrm{mg} \mathrm{BrdU} / \mathrm{kg}$ body mass or $30 \mathrm{mg} \mathrm{EdU} / \mathrm{kg}$ body mass was injected intraperitoneally once daily for 5 days. Mice were perfused 4 weeks after the last BrdU/EdU injection. EdU staining was performed with the EdU Click-It Kit (Invitrogen), following the manufacturer's instructions.

Fine olfactory discrimination. Olfactory discrimination was carried out according to a previous published protocol with minor modifications (13). Briefly, mice were separated into individual cages and deprived of water for 48 hours. Handling of an individual mouse was conducted in the home cage, consisting of the following 2 stages for each data point: a training stage and a testing stage. For the training stage, $10 \mu \mathrm{l}$ double-distilled water was placed with minimal amount $(1 \mu \mathrm{l})$ of mango extract (Mgo) to allow the mice to get used to Mgo smell. The combination of distilled water and Mgo was designated as [+], and the mice were given 2 minutes in maximum to find [+]. Once the mice finished drinking, a fresh $[+]$ solution was provided after a 30 -second interval. The amount of Mgo was increased $1.5 \mu \mathrm{l}$ per trial until it reached $8.5 \mu \mathrm{l}$. We repeated the last trial 5 times, and for the sixth trial, we presented the mice with $8.5 \mu \mathrm{l}$ almond extract (ALM) applied to the $10 \mu \mathrm{l}$ of $1 \%$ denatonium benzoate solution (Sigma-Aldrich), which was designated as [-] and is extremely bitter. The mice found it extremely aversive and learned to associate the bitter taste with the smell of ALM and avoided drinking the [-]. We conducted 4 additional trials with [-] to ensure that mice had learned the association. In the testing stage, we presented the mice with 2 dishes of water, one of which contained [+] and another containing [-]. A successful discrimination was one in which the mice drank $[+]$ and did not do the following: (a) chose [-] rather than [+]; (b) chose [+], but within 30 seconds of that selection chose [-]. If a mouse drank the [+], but within 30 seconds tasted [-] as well, we gave a half success score, distinguishing it from total failure. A total failure was assigned when a mouse tasted the [-] first. We excluded those mice from analysis that did not chose within 2 minutes. This continued for a total of 10 trials per testing stage. In additional testing sessions that decreased the content of Mgo and ALM in [+] and [-], an appropriate training stage using the new $[+]$ and $[-]$ preceded the testing stage.

AraC infusion experiments. AraC (2\%) in saline was introduced into the osmotic pump (Alzet Brain Infusion Kit 1) that was implanted under the back skin of the mice and connected to a cannula through catheter. The catheter was implanted to the lateral ventricle using a stereotaxic apparatus with the following coordinates: anterior-posterior, $0.40 \mathrm{~mm}$; mediallateral, $0.90 \mathrm{~mm}$; and dorsal-ventral, $2.15 \mathrm{~mm}$. After implantation, each mouse was housed in an individual cage for a period of 1 month before behavioral experiments were carried out. Mice were sacrificed right after behavioral testing.

Single cell culture. Primary NSPs were dissociated and 5,000 cells were cultured on poly-ornithine- and laminin-coated glass slides (5). For the $\beta$-gal experiment, these cells were cultured in NSC proliferation medium supplemented with or without WNT and WNT inhibitors $(200 \mathrm{ng} / \mathrm{ml} \mathrm{WNT3a,}$ $500 \mathrm{ng} / \mathrm{ml} \mathrm{DKK} 3$, and $100 \mathrm{ng} / \mathrm{ml}$ DKK1) for 48 hours, before being fixed with $2 \%$ PFA for 10 minutes, and subjected to $\beta$-gal staining. For differentiation experiments, cells were cultured in differentiation medium for 48 hours in the presence or absence of WNT and WNT inhibitors. Differentiated cells were fixed with $2 \%$ PFA for 20 minutes and stained with DCX and Nestin antibodies. Neuroblasts were defined as cells with small nuclei and thin processes that express DCX.

$\beta$-Gal staining. Mice were perfused and then sacrificed as previous described (5), and brains were extracted and briefly fixed for 1 hour before cryosectioning. As described previously (24), sections were $30-\mu \mathrm{m}$ thick and attached to glass slides and processed for $\beta$-gal staining. Sections were post-fixed with $4 \%$ PFA, before mounting and imaging.

Real-time RT-PCR. Tissues were frozen in dry ice, and RNA was extracted using the RNeasy Kit (Qiagen). Reverse transcription was performed using a RevertAid Kit (Fermentas) according to the manufacturer's instructions. Real-time PCR was performed using a 7000 Real-Time PCR System (Applied Biosystems) with KAPA SYBR FAST Universal qPCR Kit. Gapdh and/or $18 S$ mRNA levels were used, and each reaction was performed in triplicate for absolute quantification. The average $\mathrm{Ct}$ was determined, and experimental and control groups 
were normalized to GAPDH using the $2^{\text {-ddCt }}$ algorithm. Sequences of primers used are as follows: DKK1_F: CCTTCGGAGATGATGGTTGT, DKK1_R: GTTCTTGATCGCGTTGGAAT, DKK3_F: ATCATTGATGAAGACTGTGG, DKK3_R: CTCTTTGGAAGGCACAACAC, p21_F: GTGCAAGACAGCGACAAG, p21_R: GGAGTGATAGAAATCTGTCAG, WIP1_F: CAGAAAGGCTTCACCTCGTC, WIP1_R: CACCTCCACAGCTCTCACAA, GAPDH_F: ACCACAGTCCATGCCATCAC, and GAPDH_R: TCCACCACCCTGTTGCTGTA.

Accession numbers. All microarray data used in this study have been deposited in the Gene Expression Omnibus database with the accession number GSE57105 (mRNA microarray).

Statistics. At least 3 replicates were used in each testing group. Paired Student's $t$ tests were used for all littermate analyses. Unpaired $t$ tests were used for age-matched experiments. All $t$ tests were 2 tailed.

Study approval. All animal work was conducted under the institutional guidelines of and was approved by the Animal Ethics Committee of the Institute of Molecular and Cellular Biology.

1. Molofsky AV, et al. Increasing p16INK4a expression decreases forebrain progenitors and neurogenesis during ageing. Nature. 2006;443(7110):448-452.

2. Song H, Stevens CF, Gage FH. Astroglia induce neurogenesis from adult neural stem cells. Nature. 2002;417(6884):39-44.

3. Okamoto M, et al. Reduction in paracrine WNT3 factors during aging causes impaired adult neurogenesis. FASEB J. 2011;25(10):3570-3582.

4. Seib DR, et al. Loss of Dickkopf-1 restores neurogenesis in old age and counteracts cognitive decline. Cell Stem Cell. 2013;12(2):204-214.

5. Zhu YH, et al. WIP1 regulates the generation of new neural cells in the adult olfactory bulb through p53-dependent cell cycle control. Stem Cells. 2009;27(6):1433-1442.

6. Capela A, Temple S. LeX/ssea-1 is expressed by adult mouse CNS stem cells, identifying them as nonependymal. Neuron. 2002;35(5):865-875.

7. Choi J, Appella E, Donehower LA. The structure and expression of the murine wildtype p53induced phosphatase 1 (WIP1) gene. Genomics. 2000;64(3):298-306.

8. Pastrana E, Cheng LC, Doetsch F. Simultaneous prospective purification of adult subventricular zone neural stem cells their progeny. Proc Natl Acad Sci US A. 2009;106(15):6387-6392.

9. Goh AM, et al. Using targeted transgenic

\section{Acknowledgments}

We are very grateful to Eyleen Goh (Duke-NUS) for her recommendations regarding the AraC experiments. The research for D.V. Bulavin was supported by A*STAR (Singapore). The research for O.N. Demidov and D.V. Bulavin was supported by the Foundation ARC, France.

Received for publication January 22, 2014, and accepted in revised form April 29, 2014.

Address correspondence to: Dmitry V. Bulavin, Institute of Molecular and Cell Biology, 61 Biopolis Drive, Proteos, Singapore, 38673. Phone: 65.6586.9589; Fax: 65.6779.1117; E-mail: dvbulavin@imcb.a-star.edu.sg. Or to: Dmitry V. Bulavin, Institute for Research on Cancer and Ageing of Nice, 28, Ave de Valombrose, 06107 Nice Cedex 02, France. Phone: 33.0.492031233; Fax: 33.0.493377676; E-mail: Dmitry.Bulavin@unice.fr. reporter mice to study promoter-specific p53 transcriptional activity. Proc Natl Acad Sci U S A. 2012;109(5):1685-1690.

10. Medrano S, Burns-Cusato M, Atienza MB, Rahimi D, Scrable H. Regenerative capacity of neural precursors in the adult mammalian brain is under the control of p53. Neurobiol Aging. 2009;30(3):483-497.

11. Wong ES, et al. p38MAPK controls expression of multiple cell cycle inhibitors and islet proliferation with advancing age. Dev Cell. 2009;17(1):142-149.

12. Fujimoto $\mathrm{H}$, et al. Regulation of the antioncogenic Chk2 kinase by the oncogenic WIP1 phosphatase. Cell Death Differ. 2006;13(7):1170-1180.

13. Enwere E, Shingo T, Gregg C, Fujikawa H, Ohta S, Weiss S. Aging results in reduced epidermal growth factor receptor signaling, diminished olfactory neurogenesis, deficits in fine olfactory discrimination. JNeurosci. 2004;24(38):8354-8365.

14. Lie DC, et al. WNT signalling regulates adult hippocampal neurogenesis. Nature. 2005; 437(7063):1370-1375.

15. Tudzarova $S$, et al. Molecular architecture of the DNA replication origin activation checkpoint. EMBO J. 2010;29(19):3381-3394.

16. Muranishi Y, Furukawa T. BAC-Dkk3-EGFP transgenic mouse: an in vivo analytical tool for Dkk3 expression. J Biomed Biotechnol. 2012;2012:973140.

17. Iwano T, Masuda A, Kiyonari H, Enomoto H, Mat- suzaki F. Prox 1 postmitotically defines dentate gyrus cells by specifying granule cell identity over CA3 pyramidal cell fate in the hippocampus. Development. 2012;139(16):3051-3062.

18. Lustig B, et al. Negative feedback loop of WNT signaling through upregulation of conductin/ axin2 in colorectal liver tumors. Mol Cell Biol. 2002;22(4):1184-1193.

19. Veeck J, Dahl E. Targeting the WNT pathway in cancer: the emerging role of Dickkopf-3. Biochim Biophys Acta. 2012;1825(1):18-28.

20. Kalani MY, et al. WNT-mediated self-renewal of neural stem/progenitor cells. Proc Natl Acad Sci US A. 2008;105(44):16970-16975.

21. Barrantes Idel B, et al. Generation characterization of dickkopf3 mutant mice. Mol Cell Biol. 2006;26(6):2317-2326.

22. Qu Q, et al. Orphan nuclear receptor TLX activates $\mathrm{WNT} / \beta$-catenin signalling to stimulate neural stem cell proliferation and self-renewal. Nat Cell Biol. 2010;12(1):31-40.

23. Takai $\mathrm{H}$, et al. Chk2-deficient mice exhibit radioresistance defective p53-mediated transcription. EMBO J. 2002;21(19):5195-5205.

24. Zhu Y, Huang YF, Kek C, Bulavin DV. Apoptosis differently affects lineage tracing of Lgr 5 and Bmi1 intestinal stem cell populations. Cell Stem Cell. 2013;12(3):298-303. 